\title{
OPEN Natural phytoalexin stilbene compound resveratrol and its derivatives as anti-tobacco mosaic virus and anti-phytopathogenic fungus agents
}

\author{
Pengfei Song ${ }^{1}$, Xiuling Yu ${ }^{1 \bowtie}$, Wenqiang Yang ${ }^{1}$ \& Qingmin Wang ${ }^{2,3 凶}$
}

Plant diseases caused by plant viruses and pathogens seriously affect crop yield and quality, and it is very difficult to control them. The discovery of new leads based on natural products is an important way to innovate pesticides. Based on the resveratrol is a kind of natural phytoalexin, but it cannot be used as candidate for the development of new drug due to its poor druggability. The phenolic hydroxyl groups in the resveratrol structure are easily destroyed by oxidation, in order to improve its stability, ester formation is the most commonly used modification method in drug design. Their structures were characterized by ${ }^{1} \mathrm{H}$ NMR, ${ }^{13} \mathrm{C}$ NMR and HRMS. The activity against tobacco mosaic virus (TMV) of these ester derivatives has been tested for the first time. The bioassay results showed part of the target compounds exhibited good to excellent in vivo activities against TMV. The optimum compounds III-2 (inhibitory rates of 50,53, and $59 \%$ at $500 \mu \mathrm{g} / \mathrm{mL}$ for inactivation, curative, and protection activities in vivo, respectively), III-4 (inhibitory rates of 57,59 , and $51 \%$ at $500 \mu \mathrm{g} / \mathrm{mL}$, respectively), and II-5 (inhibitory rates of 54, 52, and $51 \%$ at $500 \mu \mathrm{g} / \mathrm{mL}$, respectively) displayed higher activity than commercial plant virucide ribavirin (inhibitory rates of 38,37 , and $40 \%$ at $500 \mu \mathrm{g} / \mathrm{mL}$, respectively). Compounds I-9 and I-10 also showed excellent activities. The systematic study provides strong evidence that these simple resveratrol derivatives could become potential TMV inhibitors. The novel concise structure provides another new template for antiviral studies.

There are many different kinds of plant viruses, which have very extensive distribution; virus diseases rank only second to the fungi categories in agricultural plant diseases. Most commercial crops are subjected to production or quality decline because of the detriment of the plant virus. Plant virus are absolute parasitic in plant cells; the required material, energy, places are provided by the host cell. Because plants do not have the complete immune system, it is difficult to prevent plant virus disease completely, therefore plant virus disease is also known as the "plant cancer"1. Tobacco mosaic virus (TMV) was the first discovered plant virus and is widely used as a model virus in the study of new antiviral agents. Its host is very wide, and it can infect many plants including capsicum, cucumber, tomato, eggplant, solanum nigrum, and so on, which brings great harm to agricultural production ${ }^{2}$. Although several commercial antiviral agents against TMV have been used, efficient and practical varieties are few. The most widely used antiviral agent ribavirin only gave an inhibition rate of no more than $50 \%$ anti-TMV effect at $500 \mu \mathrm{g} / \mathrm{mL}$, and the most effective antiviral agent ningnanmycin has an inhibitory effect of $50-60 \%$ at $500 \mu \mathrm{g} / \mathrm{mL}$. Plant diseases caused by TMV are difficult to control. Therefore, it is urgent to develop novel antiviral agents with a simple structure and outstanding activities ${ }^{3}$.

Lead discovery and optimization based on natural products are crucial means to develop novel pesticides, because their novel scaffolds can provide modes of action different from those of existing agents. Some natural products have been commercialized or used as lead compounds for plant-virus control; however, there are only a few reported economically viable antiviral chemicals available for practical application in plant protection. The

${ }^{1}$ College of Pharmacy, Linyi University, Linyi 276000, People's Republic of China. ${ }^{2}$ State Key Laboratory of Elemento-Organic Chemistry, Research Institute of Elemento-Organic Chemistry, College of Chemistry, Nankai University, Tianjin 300071, People's Republic of China. ${ }^{3}$ Collaborative Innovation Center of Chemical Science and Engineering (Tianjin), Tianjin 300071, People's Republic of China. ${ }^{\bowtie}$ email: yxlwell@163.com; wangqm@ nankai.edu.cn 
<smiles>Oc1ccc(C=Cc2cc(O)cc(O)c2)cc1</smiles>

Figure 1. Structure of resveratrol.

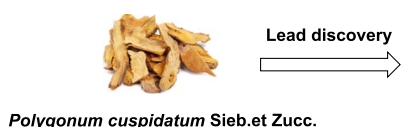

Polygonum cuspidatum Sieb.et Zucc.

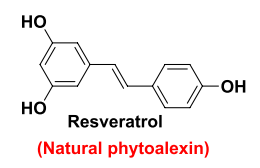

Novel antiviral agents discovery

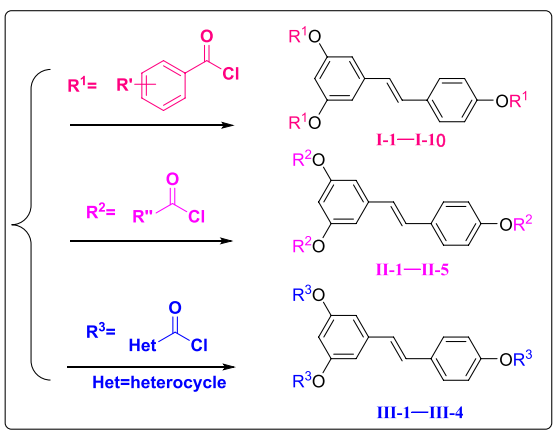

Figure 2. The design and synthesis of compounds I-1-I-10, II-1-II-5 and III-1-III-4.

naturally occurring resveratrol (3,5,4'-trihydroxy-trans-stilbene, Fig. 1) is a phytoalexin which can be activated by adverse conditions of plants, protecting against fungal infections ${ }^{4-11}$. First isolated in 1940 from Veratrum grandiflorum by Takaoka ${ }^{12,13}$, and later, it has been obtained in larger quantities from the roots of Polygonum cuspidatum $^{14}$, a plant used in traditional Chinese medicine. It attracted wider attention only in 1992 when its presence in wine was suggested as the explanation for cardioprotective effects ${ }^{15}$. Resveratrol is the most representative compound of stilbene analogues, resveratrol and its derivatives exhibits a wide range of intriguing biological activities, such as antibacterial ${ }^{16}$, antitumour ${ }^{17,18}$, antiviral ${ }^{19}$, antioxidant ${ }^{20}$, and antihypertensive activities ${ }^{21}$. Furthermore, stilbene moieties may have numerous agrochemical applications, such as in herbicides ${ }^{22-25}$. Recently, considerable attention has been focused on resveratrol derivatives. Although resveratrol possesses a series of pharmacological activities, its applications in the field of pesticide development have not been reported.

Studies have shown that modification of the structure of natural compounds can improve their biological activities. To improve the stability of resveratrol, many researchers have undertaken the synthesis and activity evaluation of resveratrol derivatives and analogues. They have modified the phenolic hydroxyl groups, double bonds and benzene ring of resveratrol so as to further understand the interactions among functional groups and its structure-activity relationship. Grow evidence indicates that this compound could be used as a lead compound in the design of drugs and resveratrol derivatives synthesized from the lead compound resveratrol or stilbene have a higher efficacy and lower toxicity ${ }^{26-29}$.

Whilst the antiviral activity of resveratrol has been extensively studied, little is known about the activity against plant viruses of resveratrol and resveratrol derivatives. Ester formation is the most commonly used modification method in drug design. A few resveratrol ester derivatives had been synthesized and tested for their antitumor activity. However, reports of the anti-TMV activity of the resveratrol ester derivatives are rather rare, and no examples are documented in the recent literature. In this paper, in order to increase the stability and druggability of resveratrol, we synthesized a series of resveratrol ester derivatives and their activities against TMV were evaluated (Fig. 2). The structure-activity relationships of these derivatives were discussed as well. Additionally, the synthesized derivatives were also investigated for their potential as fungicidal, or insecticidal agents. Resveratrol derivatives will become a research focus in future in agricultural applications.

\section{Materials and methods}

Instruments. All other commercial reagents and solvents were used as received without further purification. Reaction solvents were distilled from calcium hydride for dichloromethane and from sodium metal and benzophenone for tetrahydrofuran. E-resveratrol (98\%) was purchased from Shanxi Sciphar Hi-tech Industry Co., Ltd. (Shanxi, China). Reaction progress was monitored by thin-layer chromatography on silica gel GF254 with detection by ultraviolet (UV). Melting points were obtained using an X-4 binocular microscope melting point $(\mathrm{mp})$ apparatus and are uncorrected. Yields were not optimized. ${ }^{1} \mathrm{H}-\mathrm{NMR}$ spectra and ${ }^{13} \mathrm{C}-\mathrm{NMR}$ spectra were recorded utilizing a Bruker AV400 spectrometer with $\mathrm{CDCl}_{3}$ as solvent and tetramethylsilane as internal standard. Chemical shifts $(\delta)$ were given in parts per million $(\mathrm{ppm})$. High-resolution mass spectra (HRMS) data were obtained with a Fourier transform ion cyclotron resonance mass spectrometry (FTICR-MS) spectrometer (ionspec, $7.0 \mathrm{~T}$ ). 


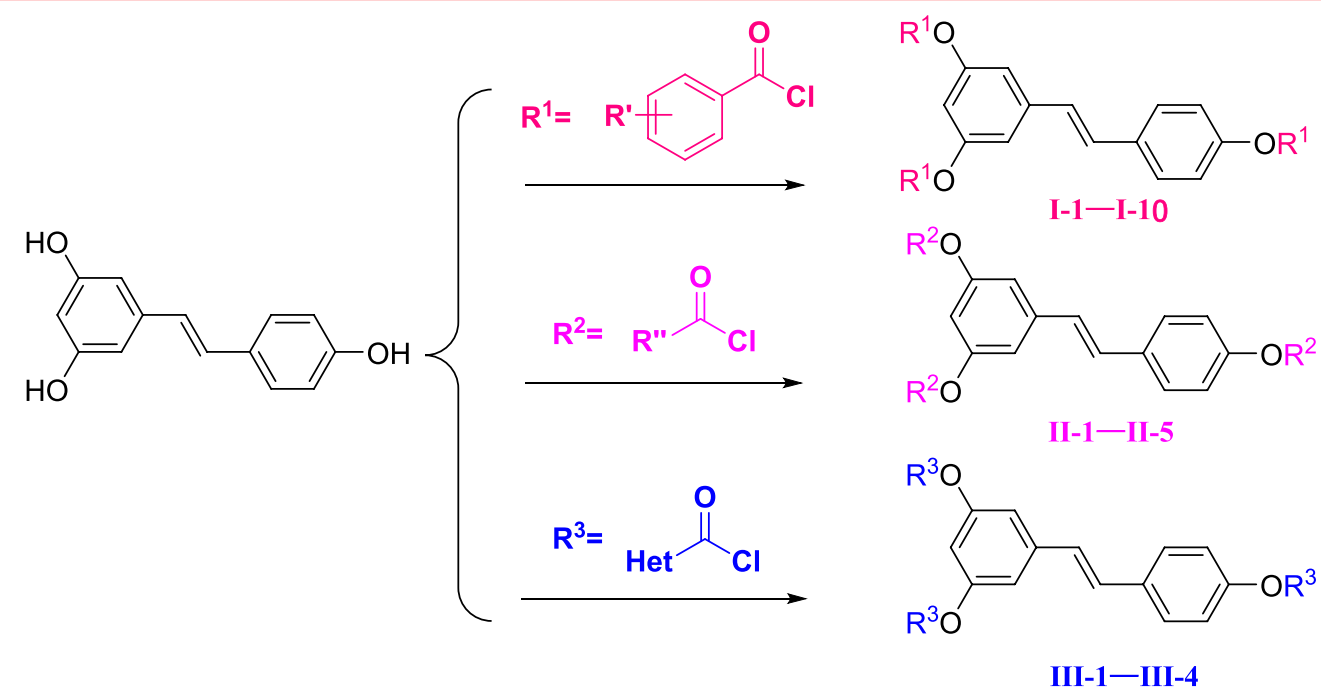

Scheme 1. The synthetic route of target compounds.

Chemical structures of I-1-I-10<smiles>[R]c1ccc(C(=O)Oc2ccc(/C=C/c3cc(OC(=O)c4ccc([R])cc4)cc(OC(=O)c4ccc([R])cc4)c3)cc2)cc1</smiles><smiles>[R]=C1CCCCC1</smiles><smiles>[R]=[W]</smiles>

I-1<smiles>[R]#Cc1ccc(C(=O)Cl)cc1</smiles>

I-3<smiles></smiles><smiles>[R]#[N+]c1ccc(C(=O)Cl)cc1</smiles>

I-5

I-6<smiles>[R]=C=CC=C(C)C(=O)Cl</smiles>

I-7<smiles>O=C(Cl)c1ccc(Cl)cc1</smiles>

I-4

$\mathbf{R}^{1}=$<smiles>Cc1cccc(C(=O)Cl)c1</smiles><smiles>[R1]=C(C)c1cc(C)c(C(=O)Cl)c(C)c1</smiles>

I-9<smiles>[R]=CC(C)(C)c1ccc(C(=O)Cl)cc1</smiles>

I-10

Scheme 2. Chemical structures of I-1-I-10.

General synthetic procedure for the target compounds I-1-I-10, II-1-II-5, and III-1-III-4 (Scheme 1). E-resveratrol $(0.1 \mathrm{~g}, 0.44 \mathrm{mmol})$ was dissolved in dichloromethane $(20 \mathrm{~mL})$, and $\mathrm{Et}_{3} \mathrm{~N}(0.22 \mathrm{~g}$, $2.19 \mathrm{mmol})$ was added. Then, $\mathrm{PhCOCl}(0.31 \mathrm{~g}, 2.19 \mathrm{mmol})$ were slowly added and the reaction mixture was stirred at room temperature. The progress of the reaction was monitored by TLC until the reaction was complete. Then the reaction mixture was added dichloromethane and water. The aqueous phase was separated and then extracted with dichloromethane twice. The combined organic layer was washed with saturated brine, dried over anhydrous sodium sulfate, and filtered. After the solvent was removed in vacuo, the residue was purified by column chromatography on silica gel to give the target compound I-1 as white solid.

Compounds I-2-I-10 (Scheme 2), II-1-II-5 (Scheme 3) and III-1-III-4 (Scheme 4) were synthesized according to the method used for compound I-1.

Data for (E)-5-(4-(benzoyloxy)styryl)-1,3-phenylene dibenzoate (I-1). White solid; yield 96\%; mp 170-172 ${ }^{\circ} \mathrm{C}$. ${ }^{1} \mathrm{H}$ NMR $\left(400 \mathrm{MHz}, \mathrm{CDCl}_{3}\right) \delta 8.22(\mathrm{~m}, 6 \mathrm{H}), 7.71-7.61(\mathrm{~m}, 3 \mathrm{H}), 7.60-7.48(\mathrm{~m}, 8 \mathrm{H}), 7.36-7.30(\mathrm{~m}, 2 \mathrm{H}), 7.15(\mathrm{~m}$, 
Chemical structures of II-1-II-5<smiles>[R]C(=O)Oc1ccc(/C=C/c2cc(OC([R7])=O)cc(OC([R7])=O)c2)cc1</smiles><smiles>[R]=C1CC1C(=O)Cl</smiles><smiles>O=C(Cl)/C=C/c1ccccc1</smiles>

II-2<smiles>[R][R]=CCC(=O)Cl</smiles>

Scheme 3. Chemical structures of II-1-II-5.

\section{Chemical structures of III-1-III-4}<smiles>[CH]C(=O)Oc1ccc(/C=C/c2cc(OC([CH])=O)cc(OC([CH])=O)c2)cc1</smiles><smiles>O=C(Cl)c1cccnc1</smiles>
$\mathbf{R}^{3}=$<smiles>O=C(Cl)c1ccsc1</smiles>

$\mathbf{R}^{3}=$<smiles>O=C(Cl)c1cccs1</smiles><smiles>[R]=C=Cc1ccoc1</smiles>

III-3

Scheme 4. Chemical structures of III-1-III-4.

$5 \mathrm{H}) ;{ }^{13} \mathrm{C} \mathrm{NMR}\left(100 \mathrm{MHz}, \mathrm{CDCl}_{3}\right) \delta 165.13,164.86,151.70,150.73,139.75,134.56,133.83,133.70,130.27,130.23$, $129.80,129.45,129.25,128.68,128.63,127.78,127.27,122.09,117.24,114.82$; HRMS (ESI) (m/z): calcd for $\mathrm{C}_{35} \mathrm{H}_{24} \mathrm{O}_{6}\left[\mathrm{M}+\mathrm{NH}_{4}\right]^{+} 558.1911$, found 558.1911.

Data for (E)-5-(4-((4-fluorobenzoyl)oxy)styryl)-1,3-phenylene bis(4-fluorobenzoate) (I-2). White solid; yield 81\%; mp 167-169 ${ }^{\circ} \mathrm{C} .{ }^{1} \mathrm{H}$ NMR $\left(400 \mathrm{MHz}, \mathrm{CDCl}_{3}\right) \delta 8.28-8.20(\mathrm{~m}, 6 \mathrm{H}), 7.56(\mathrm{~d}, J=8.0 \mathrm{~Hz}, 2 \mathrm{H}), 7.31(\mathrm{~d}, J=2.0 \mathrm{~Hz}$, 2H), 7.25-7.17 (m, 8H), 7.15-7.04 (m, 3H); $\left.{ }^{13} \mathrm{C} \mathrm{NMR} \mathrm{(100} \mathrm{MHz,} \mathrm{CDCl}_{3}\right) \delta 164.45$ (d, J=119 Hz, 1C), 164.14, 151.56, 150.62, 139.80, 134.59, 132.89 (d, J=9 Hz, 1C), 132.90, 132.80, 129.87, 127.80, 127.24, 125.47, 122.05, 117.24, 116.05, 115.97, 115.83, 115.74, 114.72; HRMS (ESI) (m/z): calcd for $\mathrm{C}_{35} \mathrm{H}_{21} \mathrm{~F}_{3} \mathrm{O}_{6}\left[\mathrm{M}+\mathrm{NH}_{4}\right]^{+} 612.1628$, found 612.1630 .

Data for (E)-5-(4-((4-methylbenzoyl)oxy)styryl)-1,3-phenylene bis(4-methylbenzoate) (I-3). White solid; yield $80 \%$; mp $159-161^{\circ} \mathrm{C} .{ }^{1} \mathrm{H}$ NMR $\left(400 \mathrm{MHz}, \mathrm{CDCl}_{3}\right) \delta 8.14-8.07(\mathrm{~m}, 6 \mathrm{H}), 7.55(\mathrm{~d}, J=8.4 \mathrm{~Hz}, 2 \mathrm{H}), 7.36-7.28$ $(\mathrm{m}, 8 \mathrm{H}), 7.24-7.02(\mathrm{~m}, 5 \mathrm{H}), 2.46(\mathrm{~s}, 9 \mathrm{H}) ;{ }^{13} \mathrm{C} \mathrm{NMR}\left(100 \mathrm{MHz}, \mathrm{CDCl}_{3}\right) \delta 165.16,164.89,151.76,150.79,144.64$, $144.50,139.66,134.50,130.29,130.26,129.73,129.38,129.32,127.72,127.30,126.72,126.54,122.10,117.16$, 114.86, 21.80; HRMS (ESI) (m/z): calcd for $\mathrm{C}_{38} \mathrm{H}_{30} \mathrm{O}_{6}\left[\mathrm{M}+\mathrm{NH}_{4}\right]^{+} 600.2381$, found 600.2379 .

Data for (E)-5-(4-((4-chlorobenzoyl)oxy)styryl)-1,3-phenylene bis(4-chlorobenzoate) (I-4). White solid; yield 97\%; mp 165-167 ${ }^{\circ} \mathrm{C} .{ }^{1} \mathrm{H}$ NMR $\left(400 \mathrm{MHz}, \mathrm{CDCl}_{3}\right) \delta 8.14-8.11(\mathrm{~m}, 4 \mathrm{H}), 8.07(\mathrm{~d}, J=8.4 \mathrm{~Hz}, 2 \mathrm{H}), 7.55-7.47(\mathrm{~m}$, 9H), $7.30(\mathrm{~d}, J=2.0 \mathrm{~Hz}, 2 \mathrm{H}), 7.21(\mathrm{~d}, J=8.4 \mathrm{~Hz}, 2 \mathrm{H}), 7.13-7.04(\mathrm{~m}, 2 \mathrm{H}) ;{ }^{13} \mathrm{C} \mathrm{NMR}\left(100 \mathrm{MHz}, \mathrm{CDCl}_{3}\right) \delta 164.24$, $163.96,161.30,151.51,150.58,141.42,140.43,140.26,139.83,134.61,131.89,131.60,129.89,129.38,129.08$, 129.00, 127.81, 122.00, 117.23, 114.62; HRMS (ESI) (m/z): calcd for $\mathrm{C}_{35} \mathrm{H}_{21} \mathrm{Cl}_{3} \mathrm{O}_{6}\left[\mathrm{M}+\mathrm{NH}_{4}\right]^{+} 660.0742$, found 660.0731 .

Data for (E)-5-(4-((4-bromobenzoyl)oxy)styryl)-1,3-phenylene bis(4-bromobenzoate) (I-5). White solid; yield 85\%; mp $183-185{ }^{\circ} \mathrm{C} .{ }^{1} \mathrm{H}$ NMR $\left(400 \mathrm{MHz}, \mathrm{CDCl}_{3}\right) \delta 8.08-8.05(\mathrm{~m}, 4 \mathrm{H}), 7.99(\mathrm{~d}, J=8.0 \mathrm{~Hz}, 2 \mathrm{H})$, 
$7.68-7.65(\mathrm{~m}, 6 \mathrm{H}), 7.55(\mathrm{~d}, J=8.4 \mathrm{~Hz}, 2 \mathrm{H}), 7.31(\mathrm{~d}, J=2.0 \mathrm{~Hz}, 2 \mathrm{H}), 7.22(\mathrm{~d}, J=8.4 \mathrm{~Hz}, 2 \mathrm{H}), 7.17-7.03(\mathrm{~m}, 3 \mathrm{H})$; ${ }^{13} \mathrm{C}$ NMR $\left(100 \mathrm{MHz}, \mathrm{CDCl}_{3}\right) \delta 164.43,164.13,161.46,151.48,150.55,139.84,134.61,132.41,132.10,132.01$, $131.95,131.70,129.90,129.18,128.08,127.82,127.20,122.01,117.25,114.63$; HRMS (ESI) (m/z): calcd for $\mathrm{C}_{35} \mathrm{H}_{21} \mathrm{Br}_{3} \mathrm{O}_{6}\left[\mathrm{M}+\mathrm{NH}_{4}\right]^{+} 793.9206$, found 793.9171.

Data for (E)-5-(4-((4-nitrobenzoyl)oxy)styryl)-1,3-phenylene bis(4-nitrobenzoate) (I-6). Pale yellow solid; yield 79\%; mp 237-239 ${ }^{\circ} \mathrm{C} .{ }^{1} \mathrm{H}$ NMR $\left(400 \mathrm{MHz}^{\mathrm{CDCl}}{ }_{3}\right) \delta 8.42-8.27(\mathrm{~m}, 12 \mathrm{H}), 7.59(\mathrm{~d}, J=8.4 \mathrm{~Hz}, 2 \mathrm{H}), 7.38(\mathrm{~d}$, $J=2.0 \mathrm{~Hz}, 2 \mathrm{H}), 7.27(\mathrm{~d}, J=8.0 \mathrm{~Hz}, 2 \mathrm{H}), 7.23-7.06(\mathrm{~m}, 3 \mathrm{H}) ;{ }^{13} \mathrm{C} \mathrm{NMR}\left(100 \mathrm{MHz}, \mathrm{CDCl}_{3}\right) \delta 162.97,151.28,151.09$, $150.39,140.11,134.79,134.46,131.40,131.37,130.95,130.20,128.86,127.97,127.15,123.89,123.80,123.72$, 121.91, 117.40, 114.37; HRMS (ESI) (m/z): calcd for $\mathrm{C}_{35} \mathrm{H}_{21} \mathrm{~N}_{3} \mathrm{O}_{12}[\mathrm{M}-\mathrm{OH}]^{-}$658.1092, found 657.9651.

Data for (E)-5-(4-((2-methylbenzoyl)oxy)styryl)-1,3-phenylene bis(2-methylbenzoate) (I-7). White solid; yield $81 \%$; mp $192-193{ }^{\circ} \mathrm{C} .{ }^{1} \mathrm{H}$ NMR $\left(400 \mathrm{MHz} \mathrm{CDCl}_{3}\right) \delta 8.21-8.15(\mathrm{~m}, 3 \mathrm{H}), 7.57(\mathrm{~d}, J=8.4 \mathrm{~Hz}, 2 \mathrm{H}), 7.52-7.47$ $(\mathrm{m}, 3 \mathrm{H}), 7.36-7.31(\mathrm{~m}, 8 \mathrm{H}), 7.22(\mathrm{~d}, J=8.4 \mathrm{~Hz}, 2 \mathrm{H}), 7.15-7.05(\mathrm{~m}, 3 \mathrm{H}), 2.70(\mathrm{~s}, 6 \mathrm{H}), 2.68(\mathrm{~s}, 3 \mathrm{H}) ;{ }^{13} \mathrm{C} \mathrm{NMR}$ $\left(100 \mathrm{MHz}, \mathrm{CDCl}_{3}\right) \delta 165.74,165.38,151.70,150.68,141.57,141.43,139.72,134.54,132.96,132.83,132.06,132.01$, 131.30, 131.21, 129.75, 128.18, 127.75, 127.29, 126.02, 125.96, 122.19, 117.26, 114.98, 22.07, 22.00; HRMS (ESI) $(\mathrm{m} / \mathrm{z})$ : calcd for $\mathrm{C}_{38} \mathrm{H}_{30} \mathrm{O}_{6}\left[\mathrm{M}+\mathrm{NH}_{4}\right]^{+}$600.2381, found 600.2379 .

Data for (E)-5-(4-((3-methylbenzoyl)oxy)styryl)-1,3-phenylene bis(3-methylbenzoate) (I-8). White solid; yield 78\%; mp $107-108{ }^{\circ} \mathrm{C} .{ }^{1} \mathrm{H}$ NMR $\left(400 \mathrm{MHz}, \mathrm{CDCl}_{3}\right) \delta 8.04-8.00(\mathrm{~m}, 6 \mathrm{H}), 7.56(\mathrm{~d}, J=8.4 \mathrm{~Hz}, 2 \mathrm{H}), 7.47-7.38$ $(\mathrm{m}, 6 \mathrm{H}), 7.32(\mathrm{~d}, J=2.0 \mathrm{~Hz}, 2 \mathrm{H}), 7.23(\mathrm{t}, J=8.0 \mathrm{~Hz}, 2 \mathrm{H}), 7.18-7.04(\mathrm{~m}, 3 \mathrm{H}), 2.46(\mathrm{~s}, 6 \mathrm{H}), 2.45(\mathrm{~s}, 3 \mathrm{H}) ;{ }^{13} \mathrm{C} \mathrm{NMR}$ $\left(100 \mathrm{MHz}, \mathrm{CDCl}_{3}\right) \delta 165.27,164.98,151.77,150.80,139.72,138.51,138.46,134.56,134.44,130.77,130.72,129.78$, 129.41, 129.23, 128.55, 128.50, 127.75, 127.42, 127.37, 127.32, 122.08, 117.17, 114.80, 21.32; HRMS (ESI) (m/z): calcd for $\mathrm{C}_{38} \mathrm{H}_{30} \mathrm{O}_{6}\left[\mathrm{M}+\mathrm{NH}_{4}\right]^{+} 600.2381$, found 600.2379 .

Data for (E)-5-(4-((2,4,6-trimethylbenzoyl)oxy)styryl)-1,3-phenylene bis(2,4,6-trimethylbenzoate) (I-9). White solid; yield 79\%; mp $173-175^{\circ} \mathrm{C} .{ }^{1} \mathrm{H}$ NMR $\left(400 \mathrm{MHz}, \mathrm{CDCl}_{3}\right) \delta 7.59(\mathrm{~d}, J=8.4 \mathrm{~Hz}, 2 \mathrm{H}), 7.33(\mathrm{~d}, J=1.6 \mathrm{~Hz}$, 2H), 7.27-7.24 (m, 2H), 7.17-7.06 (m, 3H), 6.95-6.91 (m, 6H), 2.48 (s, 12H), 2.46 (s, 6H), 2.33 (s, 6H), $2.32(\mathrm{~s}$, $3 \mathrm{H}) ;{ }^{13} \mathrm{C} \mathrm{NMR}\left(100 \mathrm{MHz}, \mathrm{CDCl}_{3}\right) \delta 168.31,167.88,151.62,150.56,140.27,140.09,139.80,135.93,135.69,134.62$, $129.85,129.51,128.79,128.70,127.86,127.33,121.95,117.04,114.26,21.23,20.24,20.07$; HRMS (ESI) (m/z): calcd for $\mathrm{C}_{44} \mathrm{H}_{42} \mathrm{O}_{6}\left[\mathrm{M}+\mathrm{NH}_{4}\right]^{+} 684.3320$, found 684.3316 .

Data for (E)-5-(4-((4-(tert-butyl)benzoyl)oxy)styryl)-1,3-phenylene bis(4-(tert-butyl)benzoate) (I-10). Pale yellow solid; yield $74 \%$; mp $102-103{ }^{\circ} \mathrm{C} .{ }^{1} \mathrm{H}$ NMR $\left(400 \mathrm{MHz}, \mathrm{CDCl}_{3}\right) \delta 8.16-8.12(\mathrm{~m}, 6 \mathrm{H}), 7.57-7.52(\mathrm{~m}, 8 \mathrm{H}), 7.30$ $(\mathrm{d}, J=2.0 \mathrm{~Hz}, 2 \mathrm{H}), 7.21(\mathrm{~d}, J=8.6 \mathrm{~Hz}, 2 \mathrm{H}), 7.14-7.02(\mathrm{~m}, 3 \mathrm{H}), 1.38(\mathrm{~s}, 18 \mathrm{H}), 1.37(\mathrm{~s}, 9 \mathrm{H}) ;{ }^{13} \mathrm{C} \mathrm{NMR}(100 \mathrm{MHz}$, $\left.\mathrm{CDCl}_{3}\right) \delta 165.11,164.86,157.62,157.49,151.78,150.80,139.66,134.51,130.18,130.14,129.74,127.74,127.30$, 126.66, 126.47, 125.67, 125.61, 122.12, 117.19, 114.87, 35.25, 31.14; HRMS (ESI) (m/z): calcd for $\mathrm{C}_{47} \mathrm{H}_{48} \mathrm{O}_{6}$ $\left[\mathrm{M}+\mathrm{NH}_{4}\right]^{+} 726.3789$, found 726.4049 .

Data for (E)-5-(4-((cyclopropanecarbonyl)oxy)styryl)-1,3-phenylene dicyclopropanecarboxylate (II-1). Yellow solid; yield $71 \%$; mp $136-138^{\circ} \mathrm{C} .{ }^{1} \mathrm{H}$ NMR $\left(400 \mathrm{MHz}, \mathrm{CDCl}_{3}\right) \delta 7.47(\mathrm{~d}, J=8.4 \mathrm{~Hz}, 2 \mathrm{H}), 7.13-6.91(\mathrm{~m}, 6 \mathrm{H})$, $6.83(\mathrm{t}, J=2.0 \mathrm{~Hz}, 1 \mathrm{H}), 1.89-1.79(\mathrm{~m}, 3 \mathrm{H}), 1.22-1.14(\mathrm{~m}, 6 \mathrm{H}), 1.07-0.99(\mathrm{~m}, 6 \mathrm{H}) ;{ }^{13} \mathrm{C} \mathrm{NMR}\left(100 \mathrm{MHz}, \mathrm{CDCl}_{3}\right)$ $\delta 173.44,173.11,151.40,150.52,139.42,134.36,129.55,127.59,127.19,121.92,116.82,114.50,13.04,9.40,9.35$; HRMS (ESI) $(\mathrm{m} / \mathrm{z})$ : calcd for $\mathrm{C}_{26} \mathrm{H}_{24} \mathrm{O}_{6}\left[\mathrm{M}+\mathrm{NH}_{4}\right]^{+} 450.1911$, found 450.1914 .

Data for (E)-5-(4-((cyclohexanecarbonyl)oxy)styryl)-1,3-phenylene dicyclohexanecarboxylate (II-2). White solid; yield 72\%; mp 131-133 ${ }^{\circ} \mathrm{C} .{ }^{1} \mathrm{H} \mathrm{NMR}\left(400 \mathrm{MHz}, \mathrm{CDCl}_{3}\right) \delta 7.47(\mathrm{~d}, J=8.4 \mathrm{~Hz}, 2 \mathrm{H}), 7.08(. \mathrm{m}, J=1.6 \mathrm{~Hz}, 3 \mathrm{H})$, $7.04(\mathrm{~d}, J=5.12 \mathrm{~Hz}, 2 \mathrm{H}), 7.00-6.91(\mathrm{~m}, 1 \mathrm{H}), 6.82-6.72(\mathrm{~m}, 1 \mathrm{H}), 2.60-2.50(\mathrm{~m}, 3 \mathrm{H}), 2.12-2.02(\mathrm{~m}, 6 \mathrm{H}), 1.87-1.78$ $(\mathrm{m}, 6 \mathrm{H}), 1.71-1.68(\mathrm{~m}, 2 \mathrm{H}), 1.65-1.55(\mathrm{~m}, 6 \mathrm{H}), 1.43-1.24(\mathrm{~m}, 10 \mathrm{H}) ;{ }^{13} \mathrm{C} \mathrm{NMR}\left(100 \mathrm{MHz}, \mathrm{CDCl}_{3}\right) \delta 174.47$, $174.11,151.56,150.65,139.44,134.34,129.55,127.58,127.20,121.88,116.73,114.42,43.22,28.94,25.72$, 25.36; HRMS (ESI) $(\mathrm{m} / \mathrm{z})$ : calcd for $\mathrm{C}_{35} \mathrm{H}_{42} \mathrm{O}_{6}\left[\mathrm{M}+\mathrm{NH}_{4}\right]^{+} 576.3320$, found 576.3323 .

Data for 5-((E)-4-(cinnamoyloxy)styryl)-1,3-phenylene (2E,2'E)-bis(3-phenylacrylate) (II-3). Yellow solid; yield $83 \%$; mp 87-89 ${ }^{\circ} \mathrm{C} .{ }^{1} \mathrm{H}$ NMR $\left(400 \mathrm{MHz} \mathrm{CDCl}_{3}\right) \delta 7.93-7.86(\mathrm{~m}, 3 \mathrm{H}), 7.62-7.57(\mathrm{~m}, 6 \mathrm{H}), 7.53(\mathrm{~d}, J=8.6 \mathrm{~Hz}$, $2 \mathrm{H}), 7.46-7.38(\mathrm{~m}, 10 \mathrm{H}), 7.25(\mathrm{~d}, J=2.3 \mathrm{~Hz}, 2 \mathrm{H}), 7.18(\mathrm{~d}, J=8.6 \mathrm{~Hz}, 2 \mathrm{H}), 7.15-6.98(\mathrm{~m}, 3 \mathrm{H}), 6.67-6.61(\mathrm{~m}, 3 \mathrm{H})$; ${ }^{13} \mathrm{C}$ NMR $\left(100 \mathrm{MHz}, \mathrm{CDCl}_{3}\right) \delta 165.36,165.04,151.52,150.57,147.07,146.80,139.62,134.51,134.16,134.11$, $130.86,130.79,129.70,129.06,128.98,128.41,128.36,127.73,127.30,121.98,117.19,117.01,114.58$; HRMS (ESI) $(\mathrm{m} / \mathrm{z})$ : calcd for $\mathrm{C}_{41} \mathrm{H}_{30} \mathrm{O}_{6}\left[\mathrm{M}+\mathrm{NH}_{4}\right]^{+}$636.2381, found 636.2383.

Data for (E)-5-(4-(propionyloxy)styryl)-1,3-phenylene dipropionate (II-4). Brown solid; yield 89\%; mp $56-58^{\circ} \mathrm{C} .{ }^{1} \mathrm{H}$ NMR $\left(400 \mathrm{MHz} \mathrm{CDCl}_{3}\right) \delta 7.48(\mathrm{~d}, J=8.4 \mathrm{~Hz}, 2 \mathrm{H}), 7.11(\mathrm{~d}, J=2.0 \mathrm{~Hz}, 2 \mathrm{H}), 7.10-7.07(\mathrm{~m}, 2 \mathrm{H})$, $7.05-6.92(\mathrm{~m}, 2 \mathrm{H}), 6.82(\mathrm{t}, J=2.0 \mathrm{~Hz}, 1 \mathrm{H}), 2.63-2.56(\mathrm{~m}, 6 \mathrm{H}), 1.27(\mathrm{t}, J=7.6 \mathrm{~Hz}, 9 \mathrm{H}) ;{ }^{13} \mathrm{C} \mathrm{NMR}\left(100 \mathrm{MHz}, \mathrm{CDCl}_{3}\right)$ $\delta 172.92,172.57,151.41,150.52,139.50,134.39,129.60,127.63,127.20,121.89,116.82,114.41,27.78,9.08,9.05$; HRMS (ESI) $(\mathrm{m} / \mathrm{z})$ : calcd for $\mathrm{C}_{23} \mathrm{H}_{24} \mathrm{O}_{6}\left[\mathrm{M}+\mathrm{NH}_{4}\right]^{+} 414.1911$, found 414.1910 .

Data for (E)-5-(4-acetoxystyryl)-1,3-phenylene diacetate (II-5). White solid; yield 65\%; mp $186-188^{\circ} \mathrm{C} .{ }^{1} \mathrm{H}$ NMR (400 MHz, $\left.\mathrm{CDCl}_{3}\right) \delta 7.48(\mathrm{~d}, J=8.5 \mathrm{~Hz}, 2 \mathrm{H}), 7.13-7.11(\mathrm{~m}, 2 \mathrm{H}), 7.10-7.07(\mathrm{~m}, 2 \mathrm{H}), 7.05-6.93(\mathrm{~m}, 2 \mathrm{H})$, 6.84-6.81 (m, 1H), $2.31(\mathrm{~s}, 9 \mathrm{H}) ;{ }^{13} \mathrm{C}$ NMR $\left(100 \mathrm{MHz}, \mathrm{CDCl}_{3}\right) \delta 169.45,169.04,151.32,150.44,139.55,134.48$, 129.67, 127.68, 127.20, 121.91, 116.93, 114.42, 21.14; HRMS (ESI) (m/z): calcd for $\mathrm{C}_{20} \mathrm{H}_{18} \mathrm{O}_{6}\left[\mathrm{M}+\mathrm{NH}_{4}\right]^{+} 372.1442$, found 372.1443 .

Data for (E)-5-(4-(nicotinoyloxy)styryl)-1,3-phenylene dinicotinate (III-1). Yellow solid; yield 71\%; mp 135-137 ${ }^{\circ} \mathrm{C} .{ }^{1} \mathrm{H}$ NMR $\left(400 \mathrm{MHz}, \mathrm{CDCl}_{3}\right)$ 89.45-9.39 (m, 3H), 8.92-8.85 (m, 3H), 8.51-8.44 (m, 3H), 7.58 (d, $J=8.4 \mathrm{~Hz}, 1 \mathrm{H}), 7.54-7.47(\mathrm{~m}, 4 \mathrm{H}), 7.37(\mathrm{~d}, J=1.6 \mathrm{~Hz}, 1 \mathrm{H}), 7.25-7.20(\mathrm{~m}, 2 \mathrm{H}), 7.17-7.14(\mathrm{~m}, 1 \mathrm{H}), 7.12-6.92$ (m, 3H); ${ }^{13} \mathrm{C}$ NMR $\left(100 \mathrm{MHz}, \mathrm{CDCl}_{3}\right) \delta 163.79,163.49,154.18,151.36,151.26,137.77,134.74,130.08,127.91$, $127.76,127.19,125.33,123.65,123.60,121.96,121.85,117.37$; HRMS (ESI) (m/z): calcd for $\mathrm{C}_{32} \mathrm{H}_{21} \mathrm{~N}_{3} \mathrm{O}_{6}[\mathrm{M}+\mathrm{H}]^{+}$ 544.1503 , found 544.1505 .

Data for (E)-5-(4-((thiophene-2-carbonyl)oxy)styryl)-1,3-phenylene bis(thiophene-3-carboxylate) (III-2). Brown solid; yield 70\%; mp 138-140 ${ }^{\circ} \mathrm{C} .{ }^{1} \mathrm{H}$ NMR (400 MHz, $\left.\mathrm{CDCl}_{3}\right) \delta 8.34-8.29(\mathrm{~m}, 3 \mathrm{H}), 7.69-7.65(\mathrm{~m}, 3 \mathrm{H})$, $7.53(\mathrm{~d}, J=8.6 \mathrm{~Hz}, 2 \mathrm{H}), 7.40-7.36(\mathrm{~m}, 3 \mathrm{H}), 7.29(\mathrm{~d}, J=2.0 \mathrm{~Hz}, 2 \mathrm{H}), 7.20(\mathrm{~d}, J=8.6 \mathrm{~Hz}, 2 \mathrm{H}), 7.16-7.01(\mathrm{~m}, 3 \mathrm{H})$; 
${ }^{13} \mathrm{C}$ NMR $\left(100 \mathrm{MHz}, \mathrm{CDCl}_{3}\right) \delta 160.96,160.65,151.42,150.47,139.70,134.54,134.35,134.17,132.78,132.56$, 129.77, 128.24, 127.76, 127.26, 126.55, 126.45, 122.06, 117.19, 114.74; HRMS (ESI) (m/z): calcd for $\mathrm{C}_{29} \mathrm{H}_{18} \mathrm{O}_{6} \mathrm{~S}_{3}$ $\left[\mathrm{M}+\mathrm{NH}_{4}\right]^{+} 576.0604$, found 576.0605 .

Data for (E)-5-(4-((thiophene-2-carbonyl)oxy)styryl)-1,3-phenylene bis(thiophene-2-carboxylate) (III-3). Yellow solid; yield 78\%; mp 113-115 ${ }^{\circ} \mathrm{C} .{ }^{1} \mathrm{H}$ NMR $\left(400 \mathrm{MHz}, \mathrm{CDCl}_{3}\right) \delta 8.03-7.96(\mathrm{~m}, 3 \mathrm{H}), 7.71-7.65(\mathrm{~m}, 3 \mathrm{H})$, $7.54(\mathrm{~d}, J=8.3 \mathrm{~Hz}, 2 \mathrm{H}), 7.34-7.29(\mathrm{~m}, 2 \mathrm{H}), 7.23(\mathrm{~d}, J=8.4 \mathrm{~Hz}, 2 \mathrm{H}), 7.20-7.16(\mathrm{~m}, 3 \mathrm{H}), 7.13-7.01(\mathrm{~m}, 3 \mathrm{H}) ;{ }^{13} \mathrm{C}$ NMR $\left(100 \mathrm{MHz}, \mathrm{CDCl}_{3}\right) \delta 160.50,160.19,151.27,150.37,139.70,134.99,134.81,134.61,133.88,133.64,132.78$, $132.52,129.84,128.15,128.09,127.76,127.25,122.01,117.22,114.62$; HRMS (ESI) (m/z): calcd for $\mathrm{C}_{29} \mathrm{H}_{18} \mathrm{O}_{6} \mathrm{~S}_{3}$ $\left[\mathrm{M}+\mathrm{NH}_{4}\right]^{+}$576.0604, found 576.0605 .

Data for (E)-5-(4-((furan-2-carbonyl)oxy)styryl)-1,3-phenylene bis(furan-2-carboxylate) (III-4). Brown solid; yield $85 \%$; mp $144-146{ }^{\circ} \mathrm{C} .{ }^{1} \mathrm{H}$ NMR $\left(400 \mathrm{MHz}, \mathrm{CDCl}_{3}\right) \delta 7.73-7.66(\mathrm{~m}, 3 \mathrm{H}), 7.53(\mathrm{~d}, J=7.9 \mathrm{~Hz}, 2 \mathrm{H})$, 7.43-7.37 (m, 3H), 7.32-7.28 (m, 2H), $7.22(\mathrm{~d}, J=8.1 \mathrm{~Hz}, 2 \mathrm{H}), 7.15-6.98(\mathrm{~m}, 3 \mathrm{H}), 6.64-6.56(\mathrm{~m}, 3 \mathrm{H}) ;{ }^{13} \mathrm{C} \mathrm{NMR}$ $\left(100 \mathrm{MHz}, \mathrm{CDCl}_{3}\right) \delta 156.83,156.46,150.92,149.99,147.46,147.27,143.89,143.68,139.76,134.65,129.88,127.81$, 127.23, 121.95, 119.87, 119.63, 117.21, 114.48, 112.32, 112.26; HRMS (ESI) (m/z): calcd for $\mathrm{C}_{29} \mathrm{H}_{18} \mathrm{O}_{9}\left[\mathrm{M}+\mathrm{NH}_{4}\right]^{+}$ 528.1289 , found 528.1291 .

Biological assay. Each bioassay was repeated three times at $25 \pm 1{ }^{\circ} \mathrm{C}$. The activity results were estimated according to a percentage scale of $0-100$ ( 0 indicating no activity and 100 indicating total mortality). The bioassay procedures for the anti-TMV, fungicidal, and insecticidal activities of the synthesized compounds are described in detailed in our published literature and can also be found in the "Supporting Information S1"30.

\section{Results and discussion}

Synthesis. In order to investigate structure-activity relationships (SARs), resveratrol was chosen as a precursor according to the in vivo anti-TMV activity listed in Table 1. Several series of resveratrol derivatives I-1-I10, II-1-II-5 and III-1-III-4 were synthesized according to procedures in Scheme 1. As shown in Scheme 1, commercially available E-resveratrol was reacted with corresponding aryl chloride, alkyl chloride and heterocyclic chloride to give resveratrol esters I-1-I-10, II-1-II-5 and III-1-III-4 in good yields. The detailed procedure is given in "Materials and methods". It's a simple route for the preparation of resveratrol derivatives with favorable yield.

Phytotoxic activity. Phytotoxic activity (according to the criterion of safety evaluation of pesticide to crops, NYT 1965.1-2010) of compounds I-1-I-10, II-1-II-5 and III-1-III-4 against tobacco was first tested. The data of phytotoxic activity at $500 \mu \mathrm{g} / \mathrm{mL}$ indicated that all of the compounds I-1-I-10, II-1-II-5 and III-1-III-4 showed no toxicity to the tested plant.

Antiviral activity. Compounds I-1-I-10, II-1-II-5 and III-1-III-4 were evaluated for their antiviral activity against TMV. The results of anti-TMV activities of these derivatives are listed in Table 1. To make a judgment on the antiviral potency of the synthesized compounds, the commercially available plant virucide ribavirin and lead compound resveratrol were used as the controls. All of the compounds were tested at both 500 and $100 \mu \mathrm{g} /$ $\mathrm{mL}$.

The synthesized compounds showed similar or higher in vivo activity against TMV than the commercial plant virucide ribavirin. At the concentration of $500 \mu \mathrm{g} / \mathrm{mL}$, resveratrol ester derivative containing meta-thienyl moieties III-2 displayed the best inhibitory effect of inactivation activity, curative activity, and protection activity with values of 50,53, and 59\%, respectively. Ribavirin as a control was studied at the same conditions with values of 38,37 , and $40 \%$, respectively. In contrast to the experimental data, the results indicated that compound III-2 was more efficient than ribavirin in vivo activity against TMV. At the concentration of $100 \mu \mathrm{g} / \mathrm{mL}$, III-2 displayed inactivation activity, 25\%; curative activity, 20\%; and protection activity, 28\%; which is higher than that of ribavirin (inactivation activity, $11 \%$; curative activity, $13 \%$; and protection activity, $12 \%$ ). Resveratrol ester derivative III-4 bearing furan groups also gave relatively higher activity $(57,59$, and $51 \%$ at $500 \mu \mathrm{g} / \mathrm{mL}$ ) than ribavirin. Especially the curative activity at $500 \mu \mathrm{g} / \mathrm{mL}$ of III-4 was higher than ribavirin and resveratrol, which indicated that the introduction of heterocycle might lead to the increase of inhibitory effect. But introduction of pyridyl (III-1) and ortho-thienyl (III-4) resulted in a decline of anti-TMV activity ${ }^{27}$. Compound II-5 with alkyl groups such as methyl resulted in an obvious improvement of anti-TMV activity which exhibited better curative and protection activities in vivo against TMV than ribavirin. More importantly, the inactivation activity of compound II-5 was much higher than ribavirin. But other derivatives bearing alkyl groups II-2, II-4, II-1, II-3 in series II showed similar or lower in vivo anti-TMV activity than ribavirin ${ }^{31}$. The introduction of aromatic substituents resulted in a decline of anti-TMV activity, however aromatic substituents of resveratrol with electron-donating groups such as methyl (I-9) or $t$-butyl (I-10) showed more excellent in vivo anti-TMV activity than ribavirin ${ }^{27}$.

Fungicidal activity. The resveratrol and its derivatives were also evaluated for their fungicidal activities with the commercial fungicides chlorothalonil and carbendazim as the controls. Resveratrol and its derivatives all exhibited fungicidal activities to some extent against 14 kinds of plant pathogens (Fusarium oxysporum sp. cucumeris; Cercospora arachidicola Hori; Physalospora piricola; Rhizoctonia cerealis; Bipolaris maydis; Colletotrichum orbiculare; Fusarium moniliforme; Alternaria solani; Fusarium graminearum; Phytophthora infestans; Phytophthora capsici; Sclerotinia sclerotiorum; Botrytis cinerea; Rhizoctonia solani) by mycelial growth method (Table 2). As shown in Table 2, target compounds exhibited broad spectrum fungicidal activities against 14 kinds of phytopathogenic fungi at $50 \mu \mathrm{g} / \mathrm{mL}$. However, compared with commercial fungicides carbendazim and chlorothalonil, these derivatives were less potent. 


\begin{tabular}{|c|c|c|c|c|}
\hline \multirow[b]{2}{*}{ Compounds } & \multirow[b]{2}{*}{ Concentration $(\mu \mathrm{g} / \mathrm{mL})$} & \multicolumn{3}{|c|}{ Relative inhibition rate (\%) ${ }^{\mathrm{a}}$} \\
\hline & & Inactivation effect & Curative effect & Protection effect \\
\hline $\mathrm{I}-1$ & 500 & $32 \pm 2$ & & \\
\hline $\mathrm{I}-2$ & 500 & $36 \pm 1$ & & \\
\hline I-3 & 500 & $34 \pm 3$ & & \\
\hline I-4 & 500 & $35 \pm 3$ & & \\
\hline I-5 & 500 & $42 \pm 4$ & & \\
\hline I-6 & 500 & $38 \pm 2$ & & \\
\hline I-7 & 500 & $37 \pm 2$ & & \\
\hline I-8 & 500 & $38 \pm 3$ & & \\
\hline \multirow{2}{*}{ I-9 } & 500 & $54 \pm 1$ & $54 \pm 4$ & $55 \pm 3$ \\
\hline & 100 & $24 \pm 1$ & $15 \pm 1$ & $20 \pm 1$ \\
\hline \multirow{2}{*}{$\mathrm{I}-10$} & 500 & $51 \pm 1$ & $56 \pm 2$ & $47 \pm 2$ \\
\hline & 100 & $21 \pm 1$ & $18 \pm 2$ & $17 \pm 2$ \\
\hline II-1 & 500 & $23 \pm 5$ & & \\
\hline \multirow{2}{*}{ II-2 } & 500 & $43 \pm 1$ & $44 \pm 4$ & $40 \pm 2$ \\
\hline & 100 & $24 \pm 2$ & $15 \pm 1$ & 0 \\
\hline II-3 & 500 & $39 \pm 3$ & & \\
\hline \multirow{2}{*}{ II-4 } & 500 & $41 \pm 5$ & $43 \pm 2$ & $46 \pm 2$ \\
\hline & 100 & $26 \pm 1$ & 0 & $25 \pm 1$ \\
\hline \multirow{2}{*}{ II-5 } & 500 & $54 \pm 1$ & $52 \pm 2$ & $51 \pm 4$ \\
\hline & 100 & $25 \pm 2$ & $28 \pm 1$ & $23 \pm 1$ \\
\hline III-1 & 500 & $30 \pm 2$ & & \\
\hline \multirow{2}{*}{ III-2 } & 500 & $50 \pm 1$ & $53 \pm 1$ & $59 \pm 4$ \\
\hline & 100 & $25 \pm 2$ & $20 \pm 2$ & $28 \pm 1$ \\
\hline III-3 & 500 & $38 \pm 3$ & & \\
\hline \multirow{2}{*}{ III-4 } & 500 & $57 \pm 1$ & $59 \pm 3$ & $51 \pm 4$ \\
\hline & 100 & $20 \pm 2$ & $26 \pm 1$ & $21 \pm 1$ \\
\hline \multirow{2}{*}{ Resveratrol $^{\mathrm{b}}$} & 500 & $50 \pm 1$ & $43 \pm 2$ & $45 \pm 2$ \\
\hline & 100 & $13 \pm 1$ & $15 \pm 1$ & $18 \pm 1$ \\
\hline \multirow{2}{*}{ Ribavirin $^{\mathrm{b}}$} & 500 & $38 \pm 2$ & $37 \pm 1$ & $40 \pm 1$ \\
\hline & 100 & $11 \pm 2$ & $13 \pm 1$ & $12 \pm 1$ \\
\hline
\end{tabular}

Table 1. The anti-TMV activity of I-1-I-10, II-1-II-5 and III-1-III-4. ${ }^{a}$ Average of three replicates. All results are expressed as the mean \pm standard deviation (SD). Prominent activity data were presented in italics. ${ }^{\mathrm{b}}$ Positive control.

Insecticidal activities. The insecticidal activities of target compounds and resveratrol against oriental armyworm (Mythimna separata), cotton bollworm (Helicoverpa armigera), corn borer (Ostrinia furnacalis), and Mosquito (Culex pipiens pallens) are listed in Table 3. The results indicated that many compounds exhibited higher activities than resveratrol. Especially compounds II-3, III-3, and III-4 exhibited obviously higher activities against oriental armyworm $(70 \%, 70 \%, 60 \%$ at $600 \mu \mathrm{g} / \mathrm{mL})$ than resveratrol.

However, though some compounds exhibited insecticidal activity on some species to some extent, the potency of these compounds as insecticide was not comparable with that of commercial insecticides. More modification on the structure should be conducted.

\section{Conclusion}

In summary, the natural product resveratrol provides an unparalleled source of inspiration for the screening of antiviral drugs. With resveratrol as lead compound by chemical modification, a series of resveratrol ester derivatives were designed and synthesized. Their activities against TMV were evaluated. The optimum compounds III-2, III-4, and II-5, I-9, I-10 displayed higher activity than commercial plant virucide ribavirin. This paper also discussed the anti-TMV activities and the structure-activity relationships of resveratrol ester derivatives, providing a reference for the development of new drugs. Resveratrol as important phytoalexin, its structure is simple and is a kind of lead compound which is extremely potential in the field of pesticide development, so the structure modification of resveratrol is a very valuable and meaningful research work. 


\begin{tabular}{|c|c|c|c|c|c|c|c|c|c|c|c|c|c|c|}
\hline \multirow[b]{2}{*}{ Compd } & \multicolumn{14}{|c|}{ Fungicidal activity $\% / 50 \mu \mathrm{g} / \mathrm{mL}^{\mathrm{a}}$} \\
\hline & $F . C^{\mathrm{b}}$ & C.H & $P . P$ & R.C & B.M & W.A & $F . M$ & A.S & F.G & P.I & P.C & S.S & R.S & B.C \\
\hline I-1 & $18 \pm 1$ & $33 \pm 1$ & $17 \pm 1$ & $26 \pm 1$ & $5 \pm 1$ & $18 \pm 2$ & $19 \pm 2$ & $43 \pm 2$ & $36 \pm 2$ & $5 \pm 1$ & $23 \pm 2$ & $40 \pm 2$ & $12 \pm 1$ & $13 \pm 1$ \\
\hline $\mathrm{I}-2$ & $14 \pm 1$ & $33 \pm 1$ & $33 \pm 1$ & $21 \pm 1$ & $5 \pm 1$ & $12 \pm 2$ & $6 \pm 1$ & $36 \pm 1$ & $36 \pm 1$ & $5 \pm 1$ & $26 \pm 2$ & $20 \pm 1$ & $16 \pm 1$ & $13 \pm 1$ \\
\hline I-3 & $18 \pm 1$ & $25 \pm 1$ & $50 \pm 1$ & $33 \pm 1$ & $27 \pm 1$ & $35 \pm 2$ & $31 \pm 1$ & $43 \pm 2$ & $21 \pm 1$ & $41 \pm 2$ & $23 \pm 1$ & $20 \pm 1$ & $48 \pm 2$ & $7 \pm 2$ \\
\hline I-4 & $5 \pm 1$ & $33 \pm 1$ & $33 \pm 1$ & $5 \pm 1$ & $14 \pm 2$ & $6 \pm 1$ & $13 \pm 2$ & $21 \pm 1$ & $14 \pm 1$ & $23 \pm 2$ & $29 \pm 1$ & $20 \pm 1$ & $15 \pm 3$ & $13 \pm 1$ \\
\hline I-5 & $9 \pm 1$ & $33 \pm 1$ & $50 \pm 1$ & $37 \pm 3$ & $18 \pm 1$ & $6 \pm 1$ & $13 \pm 1$ & $21 \pm 1$ & $27 \pm 2$ & $27 \pm 1$ & $26 \pm 2$ & $20 \pm 1$ & $31 \pm 1$ & $27 \pm 3$ \\
\hline I-6 & $5 \pm 1$ & $8 \pm 1$ & $17 \pm 2$ & $16 \pm 3$ & $9 \pm 1$ & $6 \pm 1$ & $19 \pm 3$ & $21 \pm 1$ & $36 \pm 2$ & $5 \pm 1$ & $16 \pm 1$ & $30 \pm 1$ & $16 \pm 1$ & $33 \pm 1$ \\
\hline I-7 & $5 \pm 1$ & $33 \pm 1$ & $17 \pm 2$ & $21 \pm 1$ & $14 \pm 2$ & $12 \pm 3$ & $13 \pm 2$ & $14 \pm 1$ & $14 \pm 1$ & $9 \pm 1$ & $16 \pm 1$ & $10 \pm 1$ & $25 \pm 2$ & $13 \pm 1$ \\
\hline I-8 & $14 \pm 2$ & $25 \pm 1$ & $8 \pm 1$ & $11 \pm 2$ & $14 \pm 2$ & $6 \pm 1$ & $13 \pm 1$ & $14 \pm 1$ & $36 \pm 2$ & $9 \pm 1$ & $16 \pm 1$ & $20 \pm 1$ & $20 \pm 2$ & $20 \pm 1$ \\
\hline I-9 & $5 \pm 1$ & $17 \pm 2$ & $33 \pm 1$ & $16 \pm 3$ & $18 \pm 1$ & $12 \pm 3$ & $6 \pm 1$ & $7 \pm 1$ & $36 \pm 2$ & $14 \pm 2$ & $16 \pm 1$ & $30 \pm 1$ & $5 \pm 2$ & $13 \pm 1$ \\
\hline I-10 & $5 \pm 1$ & $33 \pm 1$ & $25 \pm 1$ & $21 \pm 1$ & $14 \pm 2$ & $12 \pm 3$ & $13 \pm 2$ & $14 \pm 1$ & $21 \pm 1$ & $14 \pm 2$ & $16 \pm 1$ & $20 \pm 1$ & $15 \pm 3$ & $7 \pm 2$ \\
\hline II-1 & $5 \pm 1$ & $33 \pm 1$ & $42 \pm 3$ & $37 \pm 3$ & $17 \pm 2$ & $6 \pm 3$ & $19 \pm 2$ & $7 \pm 1$ & $36 \pm 2$ & $14 \pm 2$ & $19 \pm 1$ & $30 \pm 1$ & $31 \pm 1$ & $33 \pm 1$ \\
\hline II-2 & $14 \pm 2$ & $8 \pm 1$ & $17 \pm 2$ & $16 \pm 3$ & $5 \pm 1$ & $6 \pm 3$ & $13 \pm 2$ & $7 \pm 1$ & $21 \pm 1$ & $14 \pm 2$ & $19 \pm 1$ & $30 \pm 1$ & $20 \pm 3$ & $20 \pm 1$ \\
\hline II-3 & $18 \pm 1$ & $8 \pm 1$ & $42 \pm 3$ & $21 \pm 1$ & $5 \pm 1$ & $6 \pm 3$ & $19 \pm 3$ & $7 \pm 1$ & $7 \pm 1$ & $5 \pm 2$ & $19 \pm 1$ & $20 \pm 1$ & $31 \pm 1$ & $33 \pm 1$ \\
\hline II-4 & $18 \pm 1$ & $17 \pm 2$ & $33 \pm 1$ & $32 \pm 2$ & $18 \pm 1$ & $18 \pm 2$ & $25 \pm 1$ & $14 \pm 1$ & $7 \pm 1$ & $5 \pm 2$ & $26 \pm 3$ & $20 \pm 1$ & $21 \pm 1$ & $20 \pm 1$ \\
\hline II-5 & $5 \pm 2$ & $33 \pm 1$ & $42 \pm 2$ & $42 \pm 1$ & $18 \pm 1$ & $47 \pm 1$ & $13 \pm 1$ & $36 \pm 2$ & $36 \pm 2$ & $18 \pm 1$ & $29 \pm 1$ & $10 \pm 1$ & $36 \pm 1$ & $13 \pm 1$ \\
\hline III-1 & $14 \pm 2$ & $25 \pm 1$ & $8 \pm 1$ & $26 \pm 3$ & $14 \pm 2$ & $6 \pm 3$ & $25 \pm 1$ & $7 \pm 1$ & $7 \pm 1$ & $18 \pm 1$ & $13 \pm 3$ & $20 \pm 1$ & $31 \pm 1$ & $20 \pm 1$ \\
\hline III-2 & $14 \pm 2$ & $8 \pm 1$ & $8 \pm 1$ & $21 \pm 1$ & $5 \pm 1$ & $6 \pm 3$ & $13 \pm 2$ & $14 \pm 1$ & $21 \pm 1$ & $14 \pm 2$ & $23 \pm 2$ & $10 \pm 1$ & $15 \pm 3$ & $7 \pm 2$ \\
\hline III-3 & $5 \pm 1$ & $17 \pm 2$ & $8 \pm 1$ & $21 \pm 1$ & $14 \pm 2$ & $18 \pm 2$ & $6 \pm 1$ & $21 \pm 1$ & $36 \pm 2$ & $14 \pm 2$ & $23 \pm 2$ & $20 \pm 1$ & $16 \pm 1$ & $20 \pm 1$ \\
\hline III-4 & $14 \pm 2$ & $8 \pm 1$ & $33 \pm 1$ & $11 \pm 1$ & $5 \pm 1$ & $17 \pm 2$ & $6 \pm 1$ & $36 \pm 2$ & $36 \pm 2$ & $9 \pm 1$ & $19 \pm 1$ & $20 \pm 1$ & $12 \pm 1$ & $20 \pm 1$ \\
\hline Resveratr-ol $^{\mathrm{d}}$ & $27 \pm 1$ & $42 \pm 2$ & $25 \pm 1$ & $42 \pm 1$ & $36 \pm 1$ & $35 \pm 1$ & $13 \pm 1$ & $43 \pm 2$ & $21 \pm 1$ & $36 \pm 1$ & $42 \pm 2$ & $40 \pm 1$ & $48 \pm 1$ & $40 \pm 1$ \\
\hline Chlorothal-onil $^{c}$ & 100 & $70 \pm 1$ & 100 & $75 \pm 1$ & $<50$ & 100 & $<50$ & 100 & 100 & $92 \pm 1$ & $92 \pm 1$ & $87 \pm 1$ & 100 & 100 \\
\hline Carbenda-zim $^{c}$ & $<50$ & $<50$ & $<50$ & $<50$ & 100 & $<50$ & 100 & $<50$ & 100 & 100 & 100 & 100 & 100 & $<50$ \\
\hline
\end{tabular}

Table 2. Fungicidal activity of the compounds I-1-I-10, II-1-II-5 and III-1-III-4 against 14 kinds of phytopathogens. ${ }^{a}$ Average of three replicates. All results are expressed as the mean \pm SD. ${ }^{b}$ F.C, Fusarium oxysporium f. sp. cucumeris; C.H, Cercospora arachidicola Hori; P.P, Physalospora piricola; R.C, Rhizoctonia cerealis; B.M, Bipolaris maydis; W.A, Watermelon anthracnose; F.M, Fusarium moniliforme; A.S, Alternaria solani; F.G, Fusarium graminearum; P.I, Phytophthora infestans; P.C, Phytophthora capsici; S.S, Sclerotinia

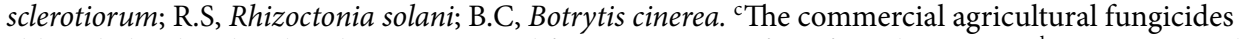
chlorothalonil and carbendazim were used for comparison of antifungal activities. ${ }^{\mathrm{d}}$ Positive control.

\begin{tabular}{|l|l|l|l|l|}
\hline Compd & Cotton bollworm $(\mathbf{6 0 0} \boldsymbol{\mu g} / \mathbf{m L})$ & Corn borer $\mathbf{6 0 0} \boldsymbol{\mu g} / \mathbf{m L})$ & Oriental armyworm $(\mathbf{6 0 0} \boldsymbol{\mu g} / \mathbf{m L})$ & Mosquito $(\mathbf{1 0} \boldsymbol{\mu g} / \mathbf{m L})$ \\
\hline I-1 & $20 \pm 2$ & $5 \pm 1$ & $20 \pm 2$ & $5 \pm 1$ \\
\hline I-2 & 0 & 0 & $10 \pm 2$ & 0 \\
\hline I-3 & $5 \pm 1$ & 0 & $10 \pm 2$ & 0 \\
\hline I-4 & 0 & 0 & $5 \pm 1$ & $20 \pm 2$ \\
\hline I-5 & 0 & 0 & 0 & 0 \\
\hline I-6 & $10 \pm 2$ & $10 \pm 2$ & $15 \pm 2$ & $5 \pm 1$ \\
\hline I-7 & 0 & $5 \pm 1$ & $10 \pm 2$ & 0 \\
\hline I-8 & $10 \pm 2$ & $15 \pm 2$ & $20 \pm 2$ & 0 \\
\hline I-9 & 0 & 0 & 0 & $5 \pm 1$ \\
\hline I-10 & $20 \pm 2$ & $10 \pm 2$ & $40 \pm 2$ & 0 \\
\hline II-1 & $5 \pm 1$ & $20 \pm 2$ & $30 \pm 2$ & $10 \pm 2$ \\
\hline II-2 & 0 & $15 \pm 2$ & $20 \pm 2$ & 0 \\
\hline II-3 & $30 \pm 2$ & $35 \pm 2$ & $70 \pm 3$ & $5 \pm 1$ \\
\hline II-4 & $10 \pm 2$ & $10 \pm 2$ & $30 \pm 2$ & $20 \pm 2$ \\
\hline II-5 & $15 \pm 2$ & $20 \pm 2$ & $50 \pm 2$ & $10 \pm 2$ \\
\hline III-1 & $5 \pm 1$ & $5 \pm 1$ & $10 \pm 1$ & $20 \pm 2$ \\
\hline III-2 & $10 \pm 2$ & $15 \pm 2$ & $40 \pm 2$ & 0 \\
\hline III-3 & $25 \pm 2$ & $35 \pm 2$ & $70 \pm 2$ & $30 \pm 2$ \\
\hline III-4 & $30 \pm 2$ & $25 \pm 2$ & $60 \pm 2$ & 0 \\
\hline Resveratrol & $5 \pm 1$ & 0 & $10 \pm 2$ & $70 \pm 2$ \\
\hline
\end{tabular}

Table 3. Insecticidal activities of the target compounds I-1-I-10, II-1-II-5 and III-1-III-4 (mortality ${ }^{\mathrm{a}}$, percent). a Average of three replicates. All results are expressed as the mean \pm standard deviation (SD). ${ }^{b}$ Positive control. 
Received: 24 April 2021; Accepted: 2 August 2021

Published online: 13 August 2021

\section{References}

1. Song, B. A., Yang, S., Jin, L. H. \& Bhadury, P. S. Environment-Friendly Anti-Plant Viral Agents 1-305 (Chemical Industry Press (Beijing) \& Springer Press, 2009).

2. Ritzenthaler, C. Resistance to plant viruses: Old issue, news answers?. Curr. Opin. Biotechnol. 16, 118-122 (2005).

3. Wei, L. Z., Meng, F. S. Antiviral composition containing fucoidan and ribavirin for preventing and treating plant viral diseases. CN 101869111A, 2010-10-27.

4. Pervaiz, S. \& Holme, A. L. Resveratrol: Its biologic targets and functional activity. Antioxid. Redox Signal 11, 2851-2897 (2009).

5. Burns, J., Yokota, T., Ashihara, H., Lean, M. E. J. \& Crozier, A. Plant foods and herbal sources of resveratrol. J. Agric. Food Chem. 50, 3337-3340 (2002).

6. Ibern-Gómez, M., Roig-Pérez, S., Lamuela-Raventós, R. M. \& Carmen, T. B. M. Resveratrol and piceid levels in natural and blended peanut butters. J. Agric. Food Chem. 48, 6352-6354 (2000).

7. Gerogiannaki-Christopoulou, M., Athanasopoulos, P., Kyriakidis, N., Gerogiannaki, I. A. \& Spanos, M. trans-Resveratrol in wines from the major Greek red and white grape varieties. Food Control 17, 700-706 (2006).

8. Fremont, L. Biological effects of resveratrol. Life Sci. 66, 663-673 (2000).

9. Bavaresco, L. et al. Effect of lime-induced leaf chlorosis on ochratoxin A, trans-resveratrol, and $\varepsilon$-viniferin production in grapevine (Vitis vinifera L.) berries infected by Aspergillus carbonarius. J. Agric. Food Chem. 56, 2085-2089 (2008).

10. Sobolev, V. S. Localized production of phytoalexins by peanut (Arachis hypogaea) kernels in response to invasion by Aspergillus species. J. Agric. Food Chem. 56, 1949-1954 (2008).

11. Soleas, G. J., Diamandis, E. P. \& Goldberg, D. M. Resveratrol: A molecule whose time has come? And gone?. Clin. Bio. Chem. 30, 91-113 (1997).

12. Jeandet, P., Bessis, R., Sbaghi, M., Meunier, P. \& Trollat, P. Resveratrol content of wines of different ages: Relationship with fungal disease pressure in the vineyard. Am. J. Enol. Vitic. 46, 1-4 (1995).

13. Takaoka, M. J. Of the phenolic substances of white hellebore (Veratrum grandiflorum Loes. Fil.). J. Fac. Sci. Hokkaido Imp. Univ. 3, 1-16 (1940).

14. Nonomura, S., Kanagawa, H. \& Makimoto, A. Chemical constituents of polygonaceous plants. I. Studies on the components of Ko-jo-kon (Polygonum cuspidatum Sieb. et Zucc.). Yakugaku Zasshi 83, 988-990 (1963).

15. Wu, C. F., Yang, J. Y., Wang, F. \& Wang, X. X. Resveratrol, botanical origin, pharmacological activity and applications. Chin. J. Nat. Med. 11, 1-15 (2013).

16. Pettit, G. R. et al. Antineoplastic agents. 487. Synthesis and biological evaluation of the antineoplastic agent 3,4-methylene dioxy5,4'-dimethoxy-3'-amino-Z-stilbene and derived amino acid amides. J. Med. Chem. 46, 525-531 (2003).

17. Nakamura, H. et al. Synthesis and biological evaluation of boronic acid containing cis-stilbenes as apoptotic tubulin polymerization inhibitors. Chem. Med. Chem. 1, 729-740 (2006).

18. Zheng, X. et al. Synthesis and anti-cancer activities of resveratrol derivatives. Open J. Med. Chem. 6, 51-57 (2016).

19. Li, Y. Q. et al. Synthesis of stilbene derivatives with inhibition of SARS coronavirus replication. Eur. J. Med. Chem. 41, 1084-1089 (2006).

20. Merillon, J. M. et al. Antioxidant activity of the stilbene astringin, newly extracted from Vitis vinifera cell cultures. Clin. Chem. 43, 1092-1093 (1997).

21. Inamori, Y. et al. Biological activities of 3,3'-dihydroxy-alpha, beta-diethylstilbene and its isomer, diethylstilbestrol. Chem. Pharm. Bull 35, 3502-3506 (1987).

22. Carella, A. et al. Synthesis and second order nonlinear optical properties of new chromophores containing 1,3,4-oxadiazole and thiophene rings. J. Chem. Soc. Perkin Trans. 2(11), 1791-1795 (2002).

23. Gester, S., Wuest, F., Pawelke, B., Bergmann, R. \& Pietzsch, J. Synthesis and biodistribution of an 18 F-labelled resveratrol derivative for small animal positron emission tomography. Amino Acids 29, 415-428 (2005).

24. Wang, X. M. et al. Two-photon pumped lasing stilbene-type chromophores containing various terminal donor groups: Relationship between lasing efficiency and intramolecular charge transfer. J. Mater. Chem. 10, 2698-2703 (2000).

25. Christian, E. et al. Covalent binding of chloroacetamide herbicides to the active site cysteine of plant type III polyketide synthases. Phytochemistry 64, 1045-1054 (2003).

26. Lu, C. J. et al. Design, synthesis, and evaluation of resveratrol derivatives as $A ß_{1-42}$ aggregation inhibitors, antioxidants, and neuroprotective agents. Bioorg. Med. Chem. Lett. 22, 7683-7687 (2012).

27. Huang, X. F. et al. Synthesis and cytotoxic evaluation of a series of resveratrol derivatives modified in C2 position. Eur. J. Med. Chem. 42, 263-267 (2007).

28. Hong, T., Jiang, W., Dong, H. M., Qiu, S. X. \& Lu, Y. Synthesis and cytotoxic activities of E-resveratrol derivatives. Chin. J. Nat. Med. 13, 375-382 (2015).

29. Moon, H. I. et al. The convenient synthesis and evaluation of the anticancer activities of new resveratrol derivatives. J. Enzyme Inhib. Med. Chem. 24, 328-336 (2009).

30. Kang, J. et al. Streptindole and its derivatives as novel antiviral and anti-phytopathogenic fungus agents. J. Agric. Food Chem. 68, 7839-7849 (2020).

31. Acerson, M. J. \& Andrus, M. B. Selective esterification of the polyphenol resveratrol at the 4'-position. Tetrahedron Lett. 55, 757-760 (2014).

\section{Acknowledgements}

This work was supported by The National Natural Science Foundation of China (no. 22007043), A Project of Shandong Province Higher Educational Science and Technology Program (no. J18KA156), Doctoral Scientific Research Foundation of Linyi University (no. LYDX2016BS097).

\section{Author contributions}

Experiment, P.S.; structure-activity relationships, W.Y.; writing-original draft preparation, X.Y. and Q.W.; writing-review and editing, P.S., X.Y. and Q.W.; supervision, Q.W.

\section{Competing interests}

The authors declare no competing interests. 


\section{Additional information}

Supplementary Information The online version contains supplementary material available at https://doi.org/ 10.1038/s41598-021-96069-1.

Correspondence and requests for materials should be addressed to X.Y. or Q.W.

Reprints and permissions information is available at www.nature.com/reprints.

Publisher's note Springer Nature remains neutral with regard to jurisdictional claims in published maps and institutional affiliations.

(c) (1) Open Access This article is licensed under a Creative Commons Attribution 4.0 International cc) License, which permits use, sharing, adaptation, distribution and reproduction in any medium or format, as long as you give appropriate credit to the original author(s) and the source, provide a link to the Creative Commons licence, and indicate if changes were made. The images or other third party material in this article are included in the article's Creative Commons licence, unless indicated otherwise in a credit line to the material. If material is not included in the article's Creative Commons licence and your intended use is not permitted by statutory regulation or exceeds the permitted use, you will need to obtain permission directly from the copyright holder. To view a copy of this licence, visit http://creativecommons.org/licenses/by/4.0/.

(C) The Author(s) 2021 